\title{
Retrofitting bacterial artificial chromosomes by homologous recombination into enhanced BAC vectors for functional studies
}

\author{
Keith Al-Hasani and Panos loannou \\ Murdoch Childrens Research Institute, University of Melbourne, \\ Royal Children's Hospital, Melbourne, VIC, Australia
}

BioTechniques 35:1054-1058 (November 2003)

The very nature of genome research demands regular improvement and development of innovative molecular tools and technologies for biotechnological and therapeutic applications. Current bacterial artificial chromosome (BAC) vector technology has enabled the construction of BAC libraries from a large number of species, including the human RPCI-11 and the mouse RPCI-23 libraries, which were used in the corresponding public sequencing genome projects. Such libraries are invaluable for an initial understanding of the genome sequence and structure of each organism and for the isolation of clones carrying most genomic loci as intact functional units. However, the majority of these libraries were made in BAC cloning vectors that do not facilitate functional studies in eukaryotic cells. To overcome this limitation, retrofitting genomic inserts as NotI fragments into novel BAC vectors has been reported (1-3). Retrofitting strategies based on targeting the insertion of a variety of cassettes into the $\operatorname{lox} \mathrm{P}$ site of P1 artificial chromosome (PAC) and $\mathrm{BAC}$ vectors by $\mathrm{Cre}$ recombinase have also been reported (4-6). Such site-specific modifications invariably depend on the insertion of a second antibiotic selection marker on the vector backbone. They also require the prior removal or inactivation of multicopy origins of replication on the retrofitting plasmids and are limited to sequences recognized by Cre recombinase. It is, therefore, not possible with these approaches to transfer genomic inserts from first generation PAC or BAC vectors into novel $\mathrm{BAC}$ vectors that are optimized for functional studies in yeast and higher organisms.

We have recently developed pEBAC190G (3), an enhanced BAC vector that combines the essential features of the first generation PAC/BAC vectors $(7,8)$ with eukaryotic elements that facilitate the transfection, episomal maintenance, and functional analysis of large genomic fragments in eukaryotic cells (9). This vector was developed primarily for the creation of new "functional" BAC libraries. However, a major barrier for the construction of novel BAC libraries is the considerable expenditure and resources required. Additionally, there are clear advantages in performing functional studies on sequenced BAC clones from the Human Genome Project rather than on new clones from functional BAC libraries, since any clones in the $100-300 \mathrm{~kb}$ range are likely to have a few hundred sequence polymorphisms with unpredictable consequences on the regulation of gene expression. The pEBAC190G vector greatly facilitates the retrofitting of genomic inserts from existing BAC libraries as Not I fragments by positive sucrose selection of recombinants (3). On the other hand, many genomic inserts in BAC clones have internal NotI sites and cannot be transferred intact to novel BAC vectors by NotI restriction digestion and ligation. Clearly, such hurdles can be overcome by partial digestion or the engineering of custom-made linkers/ adaptors for the creation of compatible sites, but such procedures can be timeconsuming.

In order to facilitate maximal use of sequenced PAC/BAC clones from the human and other genome projects, we have therefore devised a simple but effective strategy to transfer large DNA fragments from existing $\mathrm{PAC} /$ BAC vectors into our pEBAC vectors by way of vector exchange using homologous recombination. Here we demonstrate this approach for BAC clones made with the pBACe3.6 vector (10). This was achieved by exploiting the sequence homology that exists between pEBAC190G and pBACe3.6 vectors at both ends of the cloning region. The homology at one end extends over the whole length of the $s a c B$ gene $(1419 \mathrm{bp})$, while at the other end, it encompasses $41 \mathrm{bp}$ of the $s a c B$ promoter. Because both these vectors are chloramphenicol-resistant, this strategy also necessitated the replacement of the chloramphenicol cassette in pEBAC190G with kanamycin. Briefly, a 918-bp fragment encoding the kanamycin resistance gene was amplified from plasmid pCYPAC2 (7) with primers KANF 5'-CGGGCGTATTTTTTGAGTTATCGAGATTTTCAGGAGCTAAGGAAGCTAAA A TGAGCCA TATTCAACGGGAAAC-3' and KANR 5'-CAGGCGTAGCAACCAGGCGTTTAAGGGCACCAATAACTGCCTTA A A A A A A T TAGAAA A ACTCATCGAGCATC-3'. Each primer has $51 \mathrm{bp}$ at the $5^{\prime}$ end (bolded) that is homologous to sequences flanking the chloramphenicol cassette in pEBAC190G, while the sequences that are unbolded correspond to primer sequences at the $5^{\prime}$ and $3^{\prime}$ ends of the kanamycin resistance gene. About $100 \mathrm{ng}$ of the PCR product was electroporated into recombination-proficient DY380 cells (11) containing pEBAC190G, followed by selection on kanamycin/ ampicillin plates. The resulting BAC vector, pEBAC200G (Figure 1) was readily isolated and shown to have the expected structure without any other modifications or rearrangements (data not shown).

To demonstrate the utility of the pEBAC200G vector in our vector exchange protocol, we chose to transfer a 188-kb genomic fragment from a BAC clone (pBAC/265) that harbors the human FRDA locus in the pBACe 3.6 vector (12). This clone contains an internal NotI site in exon I of the FRDA locus, thus preventing its transfer as a single fragment into the pEBAC190G vector for functional studies. Preparation of the pEBAC200G vector involved the removal of the pUC-stuffer fragment by digestion with both BamHI and NotI enzymes, to reduce background 
of undigested vector, and purification of the linear fragment $(20,233 \mathrm{bp})$ by pulsed field gel electrophoresis and electroelution. Approximately $400 \mathrm{ng}$ of the linearized vector was subse- quently electroporated into recombination-proficient DY380 cells harboring pBAC/265. About 30 clones were reproducibly obtained per electroporation on $5 \%$ sucrose/kanamycin plates.

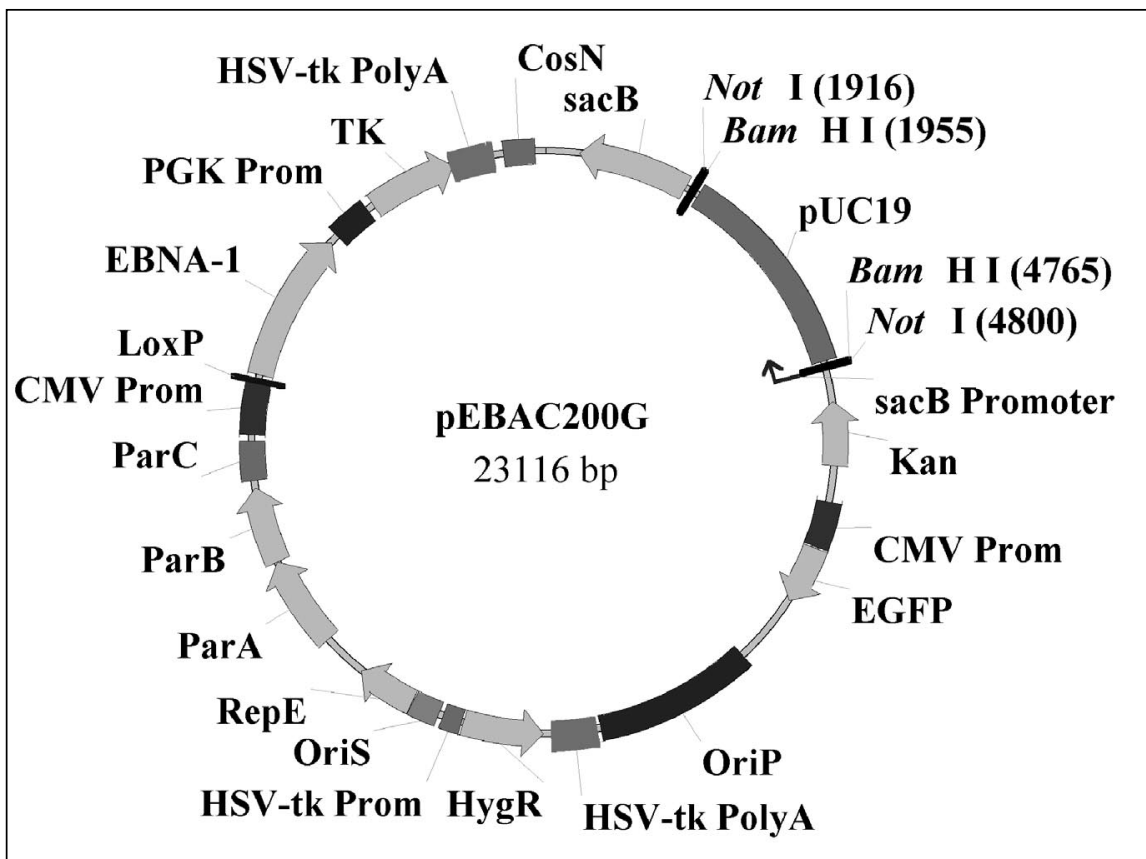

Figure 1. pEBAC200G vector. pEBAC200G was derived from the pEBAC190G (1) by the replacement of the chloramphenicol resistance gene with the kanamycin resistance gene. It has the same F plasmid origin of replication as pBeloBAC11, pBACe3.6, and other bacterial artificial chromosome (BAC) vectors, as well as the hygromycin resistance gene for selection in eukaryotic cells, the EGFP gene for identification and sorting of transfected cells, the oriP and EBNAl genes of the Epstein-Barr virus for episomal maintenance, and the thymidine kinase gene for counterselection in eukaryotic cells.

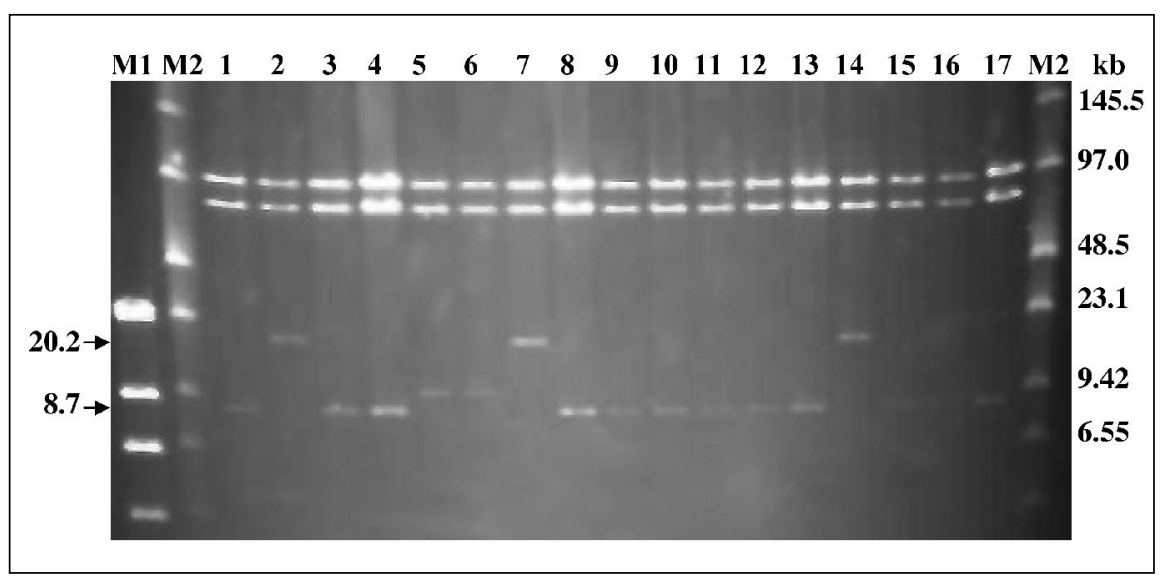

Figure 2. Vector exchange between pEBAC200G- and pBACe3.6-based bacterial artificial chromosome (BAC) clones. Swapping of the pBACe3.6 vector backbone $(8.7 \mathrm{~kb})$ in clone $\mathrm{pBAC} / 265$ from the RPCI 11 BAC library with the backbone of the pEBAC200G vector $(20.2 \mathrm{~kb})$ by homologous recombination. The purified DNA of the original (lane 1) and a recombinant clone (lane 2) was digested by Not I and analyzed by pulsed field gel electrophoresis for $12 \mathrm{~h}$ at $180 \mathrm{~V}$ and $14^{\circ} \mathrm{C}$ with initial pulse time of $2 \mathrm{~s}$ and final pulse time of $12 \mathrm{~s}$ in a $1 \%$ agarose gel. The $8.7-\mathrm{kb}$ vector band in $\mathrm{pBAC} / 265$ is replaced by the 20.2-kb pEBAC200G vector domain without any detectable change in the two genomic NotI fragments. DNA from 15 randomly picked clones after vector exchange was similarly analyzed (lanes 3-17). Correct recombinant clones (lanes 7 and 14) and clones with intermediate size vector bands (lanes 5 and 6), most likely arising from recombination at internal homology regions, were readily identified. M1, M2, $\lambda$ HindIII, and low range molecular weight markers were from New England Biolabs (Beverly, MA, USA). 
Clones generated by recombination between the linearized pEBAC200G vector and homologous sequences on the pBACe3.6 vector domain of pBAC/265 were readily identified and confirmed by pulsed field gel electrophoresis after digestion with NotI. Correct recombinant clones showed the expected shift in the length of the vector backbone from $8.7 \mathrm{~kb}$ in $\mathrm{pBAC} / 265$ (Figure 2, lane 1) to $20.2 \mathrm{~kb}$ (Figure 2, lane 2), without any detectable change in the length of the genomic Not I fragments. To understand better the nature of the false-positive clones, 15 randomly picked clones were similarly analyzed (Figure 2, lanes 3-17). Two out of 15 clones (13\%) (Figure 2, lanes 7 and 14) showed the expected change in the length of the vector, reflecting recombination at the terminal homology regions between $\mathrm{pEBAC} 200 \mathrm{G}$ and pBACe3.6. Interestingly, two clones (Figure 2, lanes 5 and 6) showed a vector band of intermediate size. Since pEBAC200G has a number of regions on the vector backbone that are identical with sequences in pBACe3.6, it is likely that clones with intermediate size vector fragments arise through recombination in internal homology regions. About $70 \%$ of the clones appeared to be false recombinants with no detectable change in the size of the vector fragment, yet extraction and re-electroporation of BAC DNA into DH10B cells showed that at least some of these clones had acquired kanamycin resistance. Such clones may arise through recombination between homologous sequences immediately flanking the chloramphenicol and kanamycin genes in the pBACe 3.6 and pEBAC200G vectors, respectively.

This approach should also be applicable to clones from BAC libraries made with the pTARBAC derivatives of the pBACe3.6 vector (http:// bacpac.chori.org/vectorsdet.htm), since the terminal homology elements that facilitate vector exchange between pBACe 3.6 and pEBAC200G are also maintained in these vectors. Vector exchange with PAC clones (kanamycinresistant) should also be possible with this approach using the pEBAC190G vector to provide selection of recombinant clones on chloramphenicol. Similar approaches may be readily adapted for the retrofitting of clones from $\mathrm{BAC}$ libraries made with the pBeloBAC11 vector (8).

In this work we have used the homologous recombination system in DY380 (11) to facilitate vector exchange. We anticipate that the GET Recombination system $(2,13)$ should also be suitable for such studies, thus obviating the need to transfer BAC clones from DH10B to DY380 cells.

In conclusion, we have developed a vector exchange approach for the convenient transfer of large genomic DNA fragments from PAC/BAC libraries made with the first generation PAC or BAC vectors into enhanced eukaryotic BAC vectors. Our strategy enables the extensive redesign and development of BAC vectors without any of the obvious limitations and restrictions of approaches that are based on site-specific recombination or the retrofitting of restriction fragments. This will greatly facilitate the incorporation of eukaryotic elements into PAC and BAC clones for functional studies and therapeutic applications.

\section{ACKNOWLEDGMENTS}

This work was supported by a grant from the Brockhoff Foundation to the Cell and Gene Therapy Research Group.

\section{REFERENCES}

1.Mejia, J.E. and A.P Monaco. 1997. Retrofitting vectors for Escherichia coli-based artificial chromosomes (PACs and BACs) with markers for transfection studies. Genome Res. 7:179-186.

2.Narayanan, K., R. Williamson, Y. Zhang, A.F. Stewart, and P.A. Ioannou. 1999. Efficient and precise engineering of a $200 \mathrm{~kb}$ beta-globin human/bacterial artificial chromosome in E. coli DH10B using an inducible homologous recombination system. Gene Ther. 6:442-447

3.Al-Hasani, K., K. Simpfendorfer, H. Wardan, J. Vadolas, F. Zaibak, R. Villain, and P.A. Ioannou. 2003. Development of a novel bacterial artificial chromosome cloning system for functional studies. Plasmid. 49: 184-187.

4.Kim, S.Y., S.K. Horrigan, J.L. Altenhofen, Z.H. Arbieva, R. Hoffman, and C.A. Westbrook. 1998. Modification of bacterial artificial chromosome clones using Cre recombinase: introduction of selectable markers for expression in eukaryotic cells. Genome Res. 8:404-412.
5.Wang, Z., P. Engler, A. Longacre, and U. Storb. 2001. An efficient method for highfidelity BAC/PAC retrofitting with a selectable marker for mammalian cell transfection. Genome Res. 11:137-142.

6.Magin-Lachmann, C., G. Kotzamanis, L. D'Aiuto, E. Wagner, and C. Huxley. 2003. Retrofitting BACs with G418 resistance, luciferase, and oriP and EBNA-1-new vectors for in vitro and in vivo delivery. BMC Biotechnol. (http://www.biomedcentral.com 1472-6750/3/2.

7.Ioannou, P.A., C.T. Amemiya, J. Garnes, P.M. Kroisel, H. Shizuya, C. Chen, M.A. Batzer, and P.J. de Jong. 1994. A new bacteriophage P1-derived vector for the propagation of large human DNA fragments. Nat. Genet. 6:84-89.

8.Shizuya, H., B. Birren, U.J. Kim, V. Mancino, T. Slepak, Y. Tachiiri, and M. Simon. 1992. Cloning and stable maintenance of 300-kilobase-pair fragments of human DNA in Escherichia coli using an F-factor-based vector. Proc. Natl. Acad. Sci. USA 89:87948797.

9.Vadolas, J., H. Wardan, M. Orford, L. Voullaire, F. Zaibak, R. Williamson, and P.A. Ioannou. 2002. Development of sensitive fluorescent assays for embryonic and fetal hemoglobin inducers using the human betaglobin locus in erythropoietic cells. Blood 100:4209-4216.

10.Frengen, E., D. Weichenhan, B. Zhao, K. Osoegawa, M. van Geel, and P.J. de Jong. 1999. A modular, positive selection bacterial artificial chromosome vector with multiple cloning sites. Genomics 58:250-253

11.Lee, E.C., D. Yu, J. Martinez de Velasco, L. Tessarollo, D.A. Swing, D.L. Court, N.A. Jenkins, and N.G. Copeland. 2001. A highly efficient Escherichia coli-based chromosome engineering system adapted for recombinogenic targeting and subcloning of BAC DNA. Genomics 73:56-65.

12.Sarsero, J.P., L. Li, H. Wardan, K. Sitte, R. Williamson, and P.A. Ioannou. 2003. Upregulation of expression from the FRDA genomic locus for the therapy of Friedreich ataxia. J. Gene Med. 5:72-81.

13.Jamsai, D., M. Orford, M. Nefedov, S. Fucharoen, R. Williamson, and P.A. Ioannou. 2003. Targeted modification of a human beta-globin locus BAC clone using GET Recombination and an I-SceI counterselection cassette. Genomics 82:68-77.

Received 21 July 2003; accepted 2 September 2003.

Address corresponding to Panos Ioannou, The Murdoch Childrens Research Institute, Royal Children's Hospital, Flemington Road, Melbourne, VIC 3052, Australia. email: ioannoup@cryptic.rch.unimelb.edu.an 